\title{
Interdisciplinary Integrated Tools to Problem Solving: A Short Course
}

\author{
Maria J. Espona \\ Argentina Information Quality (ArgIQ), Buenos Aires, Argentina
}

\begin{abstract}
Undoubtedly, we live in an information society. However, do we have the tools to deal with this situation? Since the explosion of internet, most of the world communities use the web to search for information. Some people know how to do it and other just open Google, write some words, and that is it. Then, when we try to conceptualize a problem, it is difficult to narrow down the huge amount of information to a spectrum in order to have a clear picture of it. Considering the difficulties that we saw in our students, we develop in 2014 a short course articulating systems theory, mapping studies, information quality, and competing hypothesis. Tools come from different areas of knowledge, but when used sequentially, the students go from problem conceptualization to hypothesis testing. So far, we presented it four times, with a very positive feedback of the students. In this paper, we will present our course and some of the fundamental outcomes of it.
\end{abstract}

Keywords: systems theory, mapping studies, data quality, competing hypothesis

\section{Introduction}

None has any doubt of the positive aspects of living in the information society, but most of us do not realize its dark side until we have to solve a difficult research problem, where the information available is superabundant.

The constant flux of information makes necessary to face this situation from both a comprehensive perspective and a methodologically consistent one. This is not just about the amount of information, but also its quality and how to evaluate it in order to have the optimal sources of information to work with.

Also, the lack of time to think about the definition of our research problem, how we search for information, and how we select it later creates weak points on our work that lower its academic value.

Finally, when the students are about to start to prepare their dissertation, most times, the methodology courses are too theoretic and little practical, and they give little tools to apply when it comes to write it.

Here, we will present four methodologies: systems theory, mapping studies, data quality, and competing hypothesis. All of them together allow us go from the problem conceptualization to the hypothesis testing in a methodologically consistent and structured way.

\section{Methodological Tools}

The idea of using a combination of methodological tools in an articulated way has its origin in a request made by the Peruvian Air Force. They wanted to have a dedicated course on research methodologies. However,

Maria J. Espona, M.A., professor and ArgIQ co-director, Argentina Information Quality (ArgIQ). 
for us, what has been presented to them is just a collection of isolated and non-related methodologies that has little value. Therefore, until putting on the table a series of tools, and considering along with them in the mental process we have when we are developing a research, our course come out.

Here, we will present four methodologies and show how we link them in order to give the students an easy way to apply tools.

\section{General Systems Theory}

Out of the four methods that conform to this proposal, the systemic method, developed after the general systems theory, is the oldest and widely used in different disciplines. This theory has its detractors and fervent fans that "see" reality through it.

Ludwig Von Bertalanffy, father of the general systems theory, recognized that Aristotle was actually the one who took the first step on this path with his famous phrase "the whole is greater than the sum of its parts." ${ }^{1}$ Therefore, this theory, proposed by the Austrian scientist, with an organic perspective, articulated the theoretical developments that arise in the first half of last century, on systems in different areas of knowledge.

In the book General Systems Theory, Von Bertalanffy (1969) presented the three premises that set the basis of his approach:

1. Systems exist within systems;

2. The systems are open;

3. The functions of a system depend on its structure.

Von Bertalanffy also made other contributions that dealt with the operation of these systems. For example, he suggested that systems have an input of either materials, or energy, or information, which are used within the system for its components in different processes, and then give a result or product or output.

In the above, concept is implicit. The time of the sequence input-process-output clearly indicates that we are working with a dynamic vision of reality and its components.

In order to assimilate the ideas proposed by Von Bertalanffy, we present the following examples from very different knowledge areas: biology and strategic decision-making.

The application of the systemic method to biology is so clear a familiar that surprises us-when we think about the ecosystem, a concept that it is broadly used today, we can split the word in two: "Eco"2 and "system," which means house and systems. Therefore, we can see here that the word is referring to our big house, our planet, and the relationships between its components and its evolution over time (see Figure 1).

Today, all talk about ecology, including the above concepts, without even thinking, but at the same time including it in systems thinking as to the composition and operation systems.

If we dig a little and taking the informationpresented on the subject by Food and Agriculture Organization of the United Nations (FAO) ${ }^{3}$, who when defining the concept of ecosystem, says that it "refers to the unit of animals, plants, humans, soil, climate, and all related among them and forming an ensemble in equilibrium. Each of these elements influences the other, and their relationship with each other depends on the overall balance.”

\footnotetext{
${ }^{1}$ Retrieved October 10, 2016, from http://www.goodreads.com/quotes/20103-the-whole-is-greater-than-the-sum-of-its-parts.

2 Retrieved October 10, 2016, from http://www.merriam-webster.com/dictionary/eco-(Last access, October 10, 2016).

${ }^{3}$ Retrieved May 11, 2016, from http://www.fao.org/docrep/009/ah648s/ah648s07.htm.
} 


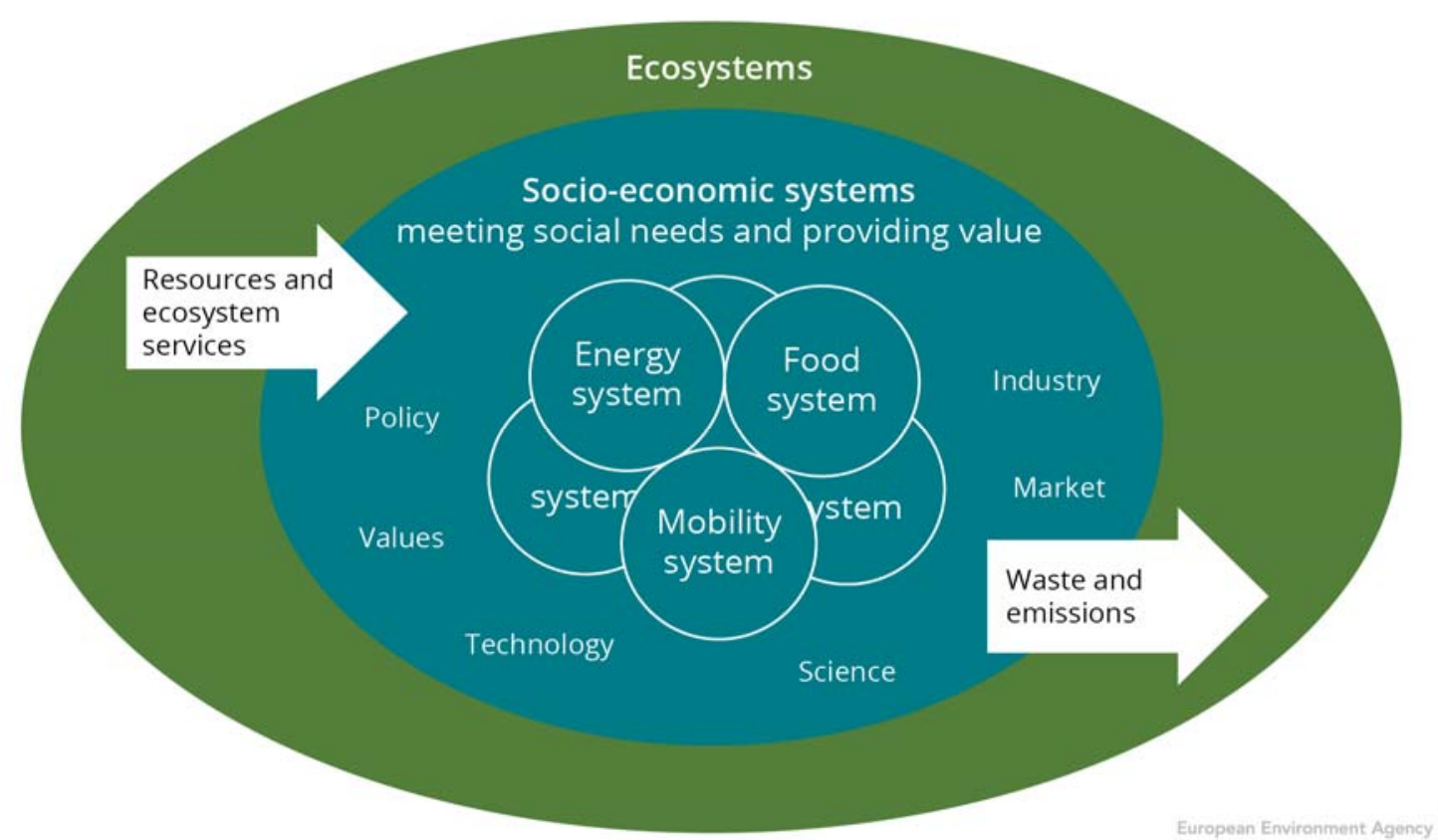

Figure 1. Ecosystems from a systemic perspective. (Source: Retrieved January 8, 2017, from http://www.eea.europa.eu/soer-2015/europe/natural-capital-and-ecosystem-services)

If we analyze the idea presented here, we see that it mentions the various components and their relationships and speaks of a balance or homeostasis, one of the emergent properties of the systems.

Then, it states that the important thing is that the very definition of the ecosystem is based on the interdependence of its components (fauna, forest, soil, water, air, and human beings), and how the balance between them can be maintained.

The example presented here shows how a theoretical development of a specific topic includes the concepts of systems theory, without even mention of it. This is the magic of this theoretical approach developed by Von Bertalanffy.

In Figure 2, we can see that a conceptual scheme of the systems theory, where its components are displayed and something is often forgotten, the border.

Given these ideas, we can draw out two types of approaches: A study of the system and its components, considering the processes that occur inside it; and another the border, characterizing and studying the processes that take place there, in the limit. In both cases, we have to consider what happens in the surroundings-from where the inputs come and to where the outputs go and why it changes.

In the specific case of our course, the systems theory is the key to take the first step in the conceptualization and understanding of the research problem.

This tool allows us to identify not only the problem we want to study, and separated from its environment by building a (theoretical or actual) border, but also individualize or identify their constitutive parts and the relationships between them and processes occurring within the system. Finally, it also allows us to define the research objectives, which, at the end, frame our system. 


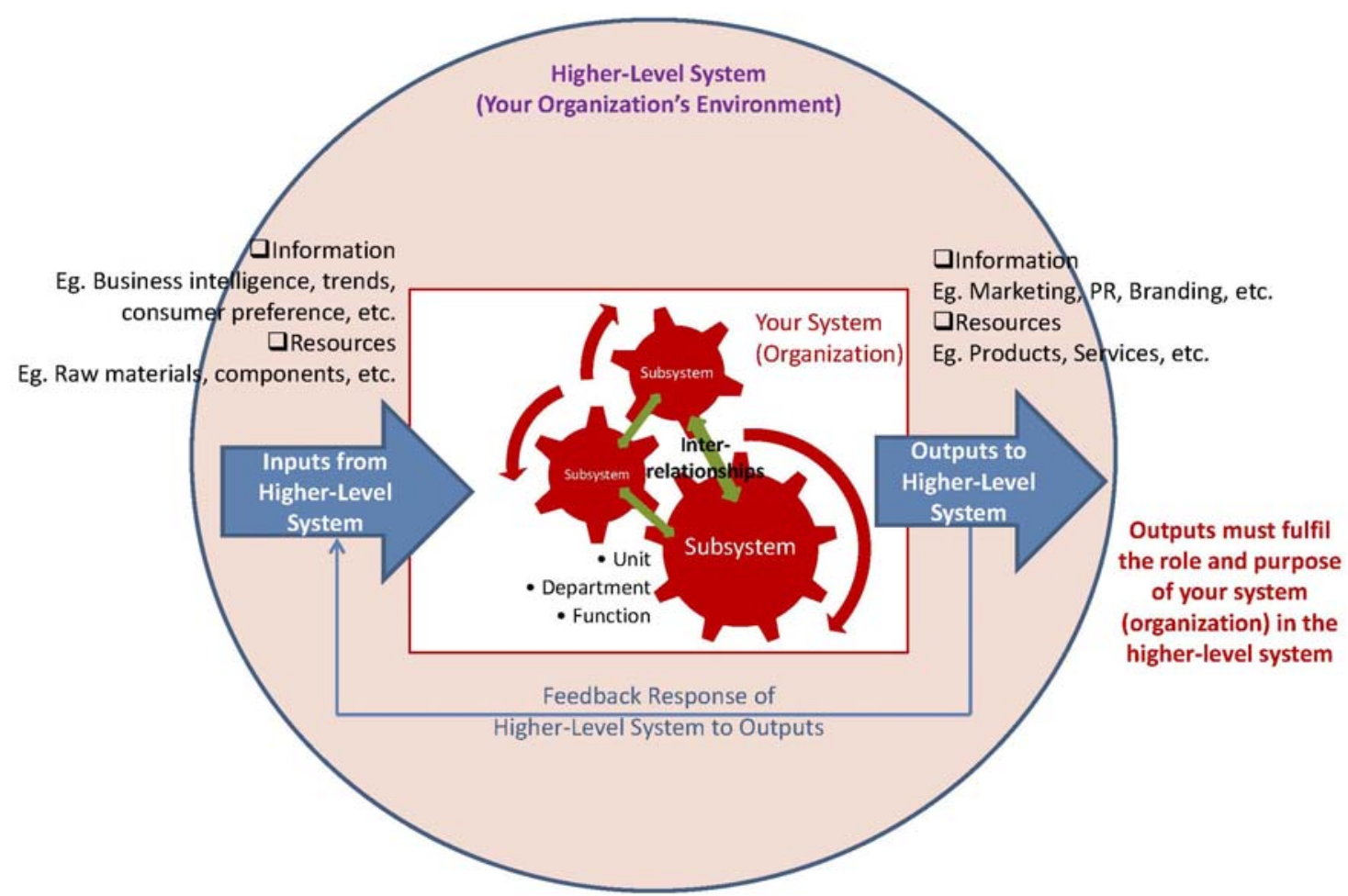

Figure 2. Systems thinking. (Source: Retrieved January 8, 2017, from http://www.hainescentreasia.com/concepts/ systems_view_of_organization.htm)

\section{Mapping Studies}

This tool comes originally from the medical sciences, where for both pharmaceutical companies and physicians themselves, it was very important to have a methodology to collect the information of the results of the implementation of certain treatments or use of a product. The collection of data should be made in such way that the gathered information should be comparable and consistent. The need of standardization was the basis for the development of this tool.

This structured search method was then discovered and assimilated by the researchers of systems engineering or informatics in general, and they mostly used it to develop the state of the art of the subjects in which they were working. Barbara Kitchencham has been the driving force behind this issue, while Dr. Marcela Genero Bocco of Alarcos Group (UCLM, Spain) is the leading Spanish-speaking experts ${ }^{4}$.

But before going into detail of this tool, it is important to understand the reason for their inclusion here.

When most people perform searches, especially when rigor is required in the collection and analysis of results, many times, we just select a search engine, either Google or some virtual library, then we enter some keywords, and finally we review the results and choose some of them. We follow this sequence of steps almost without thinking, and without registering the process or the results or the selection criteria.

This way of working smuggles many errors, either by the keywords we entered into the search engine, or the articles selection criteria. In the latter case, many times our cognitive biases make us choose based on whether it is useful to our research, or comes from a source we consider valid. In other words, if its supports

\footnotetext{
${ }^{4}$ This section was developed using the chapter on systematic literature reviews of the book she coauthored Métodos de investigación en ingeniería del software (Research methods in software engineering).
} 
our thinking, we select it. We have this tendency, and unless we use a tool that allows us to avoid such a deeply rooted bias, we are lost even before we start!

This is where the systematic review comes to our aid, giving us a guide of standardized procedures, with checkpoints, which allows us to perform a structured and replicable search.

Here is the sequence of work, divided into three phases.

Phase one: Planning the review. The first step is to identify the need for a review. It has to do with the need to summarize existing relevant information on a topic or research problem. Having that ensemble of information allows us to know the state of art of an issue or problem.

The second step is to formulate research questions. This is the most important part, since they will lead the search process. Here we take what developed in the systems theory stage, when we study and conceptualize our problem, and then, identify what aspects of it are going to study and how we will do it. The formulations of the research objectives and research questions are the links between these first two methodologies.

A key point of this tool is that when the search is done, the selection of articles will be carried out based on whether they answer or not to the research questions, not on how they do. This is how we start to elude our cognitive biases.

Once, we have questions, we need to define the search protocol-a formal and detailed specific plan, where each of the steps and features are identified. There is a list of the components of our search strategy:

(a) search terms, alternative terms, and synonyms (use OR and AND);

(b) sources of information (use virtual libraries, magazines, conferences, gray literature, or Google);

(c) inclusion and exclusion criteria;

(d) quality criteria of each publication (of its content regarding the research problems and objectives);

(e) strategy for data extraction (form design);

(f) synthesis of extracted data (data analysis form in relation to the research questions).

Phase two: Executing the review. Now, we do the search work itself-identify relevant research using selected search terms and apply the criteria of inclusion and exclusion on the results obtained after using the chosen search engine.

It is important to point out that even when this tool was designed to be used both in medical search and later in academic libraries, its use by other search engines like Google was proved to be very useful and generated interesting results. This aspect is quite important, if we consider that not all the students have access to a digital library and that not all problems are academic ones.

Once, the first search is done we can analyze if our original protocol needs a revision, for example, refine or expand the keywords, change the research questions, or use other sources of information, in order to have relevant and representative articles to be analyzed.

If we consider that, the results obtained are fine, we can proceed with the selection of primary studies by first applying the criteria of inclusion and exclusion to all articles retrieved, and second, those who remain are studied using the research questions. Those answers are incorporated on the data extraction file (usually an excel file), which already contains data specific to each publication (author, date, publisher, title, etc.). Then, these results are analyzed in a traditional way, and if feasible, graphics are produced in order to better show the results of the mapping study.

5 “OR" and “AND” are operators (“or” and “and”) in the search. 
Not always a study of the quality of selected studies is done. Its importance will depend on the subject and the particular case. In any case, we must specify the criteria and method of evaluation, whether qualitative or quantitative.

Phase three: Writing the report. In this last phase, the results obtained are shown and it is essential to clearly state the procedure followed, including every possible detail of our work. The purpose of this way of presenting information is to provide reader with all the information he/she could need to repeat our study.

In the case of our methodological integration, the performance of the systematic review is essential for the other two tools that follow. At first, the raw material will be evaluated in terms of their quality with the methodology of information quality, and then, they will be the evidence used to validate the assumptions with the methodology of competing hypotheses.

\section{Information Quality Core Concepts ${ }^{6}$}

In a world with an overload of information, it is important to find a method to evaluate and categorize the information in order to better profit both its content and metadata.

In this context, the method information quality, developed by researchers at the Massachusetts Institute of Technology (MIT) (Cambridge, Massachusetts, USA), provides help.

We consider their methodology as a three legs table: categories and dimensions, participants, and the total data quality management (TDQM) cycle.

Categories and dimensions. Wang and Strong (1996) developed a framework to evaluate and hierarchically organize information. In order to build it, they survey information consumers and Master in Bussiness Administration (MBA) students about which attributes the information should have, resulting in 179 attributes. Then, they did a new survey with the objective of learning the importance of the attributes identified before to a bigger pool of data consumers. At the end of the process, they identify 16 dimensions, grouped into four categories (see Table 1).

Table 1

Information Quality Categories and Dimensions (Source: Fisher, Lauria, Chengalur-Smith, \& Wang, 2006)

\begin{tabular}{|l|l|}
\hline Categories & Dimensions \\
\hline Intrinsic IQ & Accuracy, believability, objectivity, and reputation \\
\hline Contextual IQ & Value-added, relevancy, timeliness, completeness, and amount of data \\
\hline Representational IQ & Interpretability, ease of understanding, representational consistency, and conciseness of representation \\
\hline Accessibility IQ & Access and security \\
\hline
\end{tabular}

Note. ${ }^{*} \mathrm{IQ}$ is the abbreviation of intelligence quotient.

Participants. The participants are collector, custodian, and consumer (3C's), who are the stakeholders in the flow of information inside organizations. The identification of the role played by the different actors in a given process and an evaluation of their performance will allow the understanding of organizational problems.

TDQM. TDQM is a continuous cycle that when applied by organizations, it will allow them to implement an IQ Program and check its results in the future. It includes four steps: Definition, measurement, analysis, and improvement.

\footnotetext{
${ }^{6}$ This section is an extract of the Espona and Fisher article presented at ISECON 2015: Teaching information quality to professionals in intelligence government agencies.
} 


\section{Competing Hypothesis}

This tool was developed by Richards J. Heuer Jr., an intelligence analysis expert from the Central Intelligence Agency (CIA), USA, between 1978 and 1986, and was released for the entire community in his book Psychology of Intelligence Analysis.

This methodology was designed to be used in cases of complex problems, where there are many possible futures scenarios and there are many evidences to be considered for their verification.

The operating mechanism of this tool is the simultaneous study of all possible hypothesis and contrasting them with existing evidence. The result will be a table with hypothesis organized according to amount and type of evidence each one has (see Table 2).

The hypothesis matrix shows the estimated correlation between the two elements (evidences and hypothesis), and by the sum of the different probabilities, it shows which hypothesis has more support, considering the evidence available.

It is important to point out what Heuer (1999) said, "The result of the methodology is which hypothesis has more support according with the available evidence and not which is the hypothesis with higher probability of occurrence."

In the context of our methodological integration, we generate the hypotheses using our research questions from the mapping study.

The evidence used here is the one identified in the stage of systematic review, which is also evaluated with the tool of information quality. It is also possible to analyze how behave the evidence according to its quality hierarchy with the hypothesis we are testing.

Also, using as evidence - the items obtained in the review—we avoid, in a very important way, cognitive biases that would have us choose only confirmatory evidence of different scenarios. Table 2 shows the format of the matrix is observed.

Table 2

Competing Hypothesis Matrix (Source: Heuer, 1999)

\begin{tabular}{lll}
\hline & Hypothesis1 & Hypothesis 2 \\
\hline Evidence 1 & $+{ }^{*}$ & + \\
Evidence 2 & + & -- \\
Evidence 3 & + & Not apply \\
Evidence 4 & Not apply & ++ \\
Evidence 5 & $-^{*}$ & + \\
Total & $3+, 1-$ & $4+, 2-$ \\
\hline
\end{tabular}

Note. The ${ }^{*}+$ means the evidence support the hypothesis and how strong it does. The *- means that it does not support it and not apply means that has not relation to the hypothesis under study.

\section{The Course}

Under the umbrella of the Humboldt International University of Miami (HIU) (http://www.hiuniversity.com) and the Non Govermental Organization (NGO) Argentina Information Quality (ArgIQ) (http://www.argiq.com.ar), we present this course four times.

Here, we present the four tools that are part of this methodological proposal, which aim to help the researcher to work in an environment of high volume of information, high uncertainty, and cognitive biases. Nevertheless, it is worth to mention that, at the beginning of the course, we took some time to try to understand 
how we think and how we make decisions, with the objectives of making the students reflect on their cognitive biases and remind them of how to deal with them.

Integration works as follows:

1. The systems theory helps us to frame the research problem while identifying the objectives we intend to work with;

2. The previously developed objectives are transformed into research questions for the mapping study. This methodology is critical because it will be used to identify and select the items we use as raw material for our work;

3. The articles selected in the mapping study will be evaluated and hierarchically organized according to the information quality categories and dimensions;

4. At the final stage, the research questions are used to develop the hypothesis, and the items selected in the mapping study will be the evidence (hierarchically organized according to the information quality tool) used to test each hypothesis.

The students should write at the end a document where all the tools are integrated, and show their conclusions at the end.

During the course, we work intensively with students and help them to think and analyze their own research problem using each methodology.

So far, we presented this course in two formats: In the traditional class and use of a website to exchange material and send tasks and interact between classes. Depending on the students, each of the modalities had its own pros and cons.

Until today, we presented the course four times with different results, and most of them are positives. The students consider that the use of the enchained methodological tools help them to finally "solve" their research problem and give them a new perspective, when it comes to read other kind of documents or thesis.

The sole negative results were obtained, when the students did not have a research problem to work with. They learnt how to use the tools, but they never arrived to a final document with conclusions.

Out of the 43 students that complete the course, just about 15 are willing to rewrite their final document into a book chapter that is to be published by the HIU, and it will be used as course material.

\section{Conclusion}

With the experience that we have so far, we can say that the tools we develop and present here is quite useful for our students, not just to deal with a typical academic research problem, but also to evaluate others research and developments.

They were able to incorporate all the methodologies and learned how to apply them in different problems.

What we consider most important is that they were able at the end of the course to deal with their cognitive biases.

\section{References}

Espona, M. J. (2016). Metodologías para el análisis de información: Una necesidad de nuestros días (Metodologies for information analysis: An our day need). In F. G. Javier, D. L. Teresa, \& O. Eduardo (Eds.), Desarrollo de competencias para el siglo XXI (Competencies development for the 21st century). Miami, Florida: Alexandria Library Publishing House.

Fisher, C., Lauria, E., Chengalur-Smith, I., \& Wang, R. Y. (2006). Introduction to information quality. Cambridge, Massachusetts: Massachusetts Institute of Technologi Information Quality (MITIQ). 
Food and Agriculture Organization of the United Nations (FAO), Spanish. (n.d.). Introduction to ecology. Retrieved January 8, 2017, from http://www.fao.org/docrep/009/ah648s/ah648s07.htm

Genero, M., Cruz-Lemus, J. A., \& Piattini, M. (2014). Métodos de investigación en ingeniería del software (Software engeneering research methods) (pp. 199-246). RaMa: Madrid.

Heuer, R. (1999). Psychology of intelligence analysis. Washington, D.C.: Center for the Study of Intelligence. Retrieved March 3, 2015, from http://www.cia.gov/library/center-for-the-study-of-intelligence/csi-publications/books-and-monographs/ psychology-of-intelligence-analysis/PsychofIntelNew.pdf

Kitchencham, B., Rialette, P., Budgen, D., Brereton, P., Turner, M., Niazi, M., \& Linkman, S. (2007). Guidelines for performing systematic literature reviews in software engineering. Information and Software Technology, 52(2010), 792-805. Retrieved January 8, 2017, from http://www.cs.auckland.ac.nz/ mria007/Sulayman/Systematic_reviews_5_8.pdf

Von Bertalanffy, L. (1969). General systems theory: Foundations, development and applications (p. 289). Retrieved January 8, 2017, from http://monoskop.org/images/7/77/Von_Bertalanffy_Ludwig_General_System_Theory_1968.pdf

Wang, R. Y., \& Strong, D. (1996). Beyond accuracy: What data quality means to data consumers. Journal of Management Information Systems, 12(4), 5-34. 